\title{
Scaling laws for rotating Rayleigh-Bénard convection
}

\author{
J. D. Scheel* and M. C. Cross \\ Department of Physics, California Institute of Technology 114-36, Pasadena, California 91125, USA
}

(Received 13 June 2005; published 14 November 2005)

\begin{abstract}
Numerical simulations of large aspect ratio, three-dimensional rotating Rayleigh-Bénard convection for no-slip boundary conditions have been performed in both cylinders and periodic boxes. We have focused near the threshold for the supercritical bifurcation from the conducting state to a convecting state exhibiting domain chaos. A detailed analysis of these simulations has been carried out and is compared with experimental results, as well as predictions from multiple scale perturbation theory. We find that the time scaling law agrees with the theoretical prediction, which is in contradiction to experimental results. We also have looked at the scaling of defect lengths and defect glide velocities. We find a separation of scales in defect diameters perpendicular and parallel to the rolls as expected, but the scaling laws for the two different lengths are in contradiction to theory. The defect velocity scaling law agrees with our theoretical prediction from multiple scale perturbation theory.
\end{abstract}

DOI: 10.1103/PhysRevE.72.056315

PACS number(s): 47.54.+r, 47.20.Bp, 47.32.-y, 47.27.Te

\section{INTRODUCTION}

Rayleigh-Bénard convection is a paradigmatic system for studying pattern formation [1]. This system exhibits weak turbulence, otherwise known as spatiotemporal chaos. If the bifurcation to chaos is smooth, then we can study the scaling laws near such a transition.

In Rayleigh-Beńard convection, a fluid cell bounded by horizontal parallel plates is kept at a constant temperature difference $\Delta T$. This leads to a buoyancy-driven instability as $\Delta T$ increases past a critical value. In nonrotating RayleighBénard convection, the conducting state bifurcates to a convection state consisting of straight, parallel rolls. Then if the aspect ratio is large, or if $\Delta T$ is increased further, these states can bifurcate to spatiotemporally chaotic states. The parameter regime where parallel rolls are stable is well modeled theoretically near threshold by a stability analysis of the nonlinear state $[2,3]$.

In rotating convection, the entire cell is rotated about a vertical axis with a constant rotation rate $\Omega_{D}$, which breaks the reflection symmetry. For a certain range of parameters, a chaotic state known as domain chaos has been found to exist, as shown in Fig. 1. This state consists of domains of parallel rolls, each of whose particular location and size vary chaotically. A perturbation expansion of the equations which model rotating Rayleigh-Bénard convection [4,5] predicted this supercritical bifurcation from a conducting state to an unstable convection state. A supercritical bifurcation is particularly useful to study since the new state evolves continuously out of the old state. A control parameter $\epsilon$ [Eq. (4)] can characterize this transition. This control parameter measures the strength of the driving above the onset of convection, and is linear in the temperature difference.

In the original work by Küppers and Lortz [4], the convection state consisted of straight parallel rolls which were unstable to rolls at a different, distinct orientation. For large Prandtl number $(>10)$, this orientational angle is about $60^{\circ}$

*Electronic address: jscheel@caltech.edu with respect to the original. This is known as the KüppersLortz instability, and can be seen in Fig. 2, where the dominant roll orientation is plotted as a function of time for simulations in a periodic geometry. After about 400 vertical diffusion times (enough to allow the transients to relax), the orientation of the rolls switches discretely. However, one can see that the time between switches gets longer and longer. ${ }^{1}$ This is completely consistent with the predictions of Busse and Heikes [6], who noted that the time between roll switches should increase as the perturbation which causes the instability decays.

However, in experimental systems, one typically sees a more constant switching frequency such as that seen in Fig. 3, which is for the same system as Fig. 2, but for larger $\epsilon$. Busse and Heikes suggested thermal or other extrinsic noise would cause the roll switches to occur at a relatively constant rate. Laveder et al. [7] demonstrated that a noise floor would also have a significant effect on the scaling laws for correlation lengths. However, Oh and Ahlers showed [8] that the experimental noise in Rayleigh-Bénard convection systems is very small. It is practically insignificant in the parameter range investigated by the experiments of $\mathrm{Hu}$, et al. Cross and $\mathrm{Tu}$ [9] showed that in realistic systems the chaotic fluctuations from a complex spatial structure are important, leading to roll switches occurring at a relatively constant rate.

This led to theoretical modeling of rotating RayleighBénard convection via three coupled Ginzburg-Landau equations [9-11], which confirmed the presence of a supercritical bifurcation from the conducting state to a domain chaotic state as the temperature difference is increased at fixed rotation rate (but above some critical rotation rate). The patterns switched at a constant rate and looked similar to those seen in experiments. The theory uses periodic boundary conditions and assumes that the dynamics is dominated by domain wall motion. When one set of rolls is replaced by another, the region containing the new, growing set of rolls spreads by

\footnotetext{
${ }^{1}$ This slowing down is seen for the duration of our simulation. It is possible that if the simulation is run out for longer the switching time will become constant.
} 


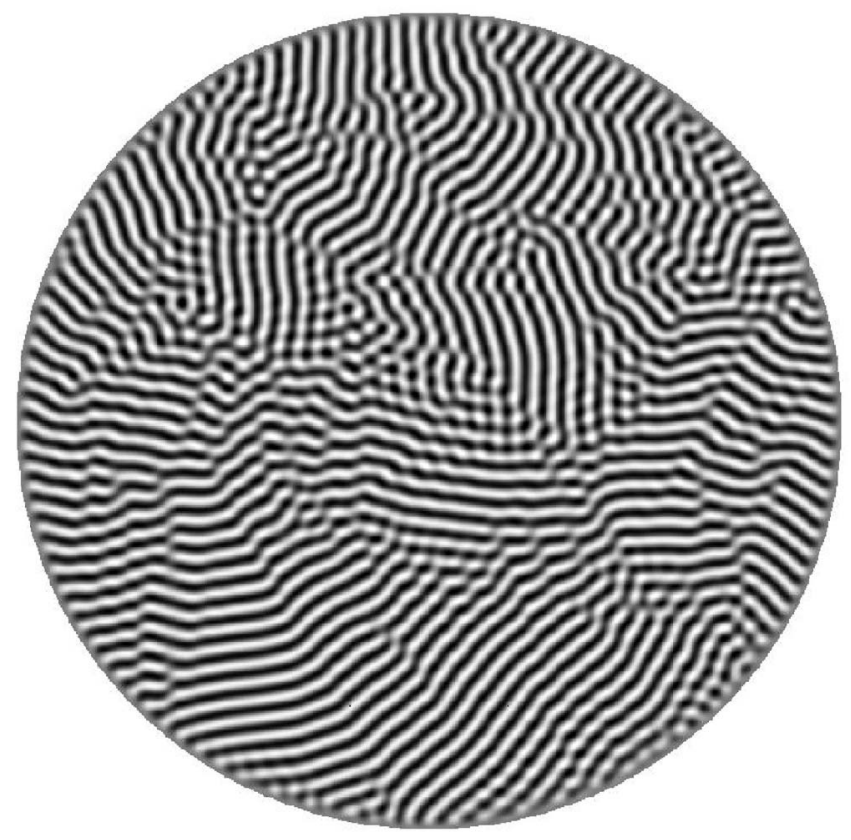

FIG. 1. Snapshot of temperature deviation $\theta$ at the midplane, for a domain chaos state with the following parameters: $\Gamma=40, \sigma$ $=0.93, \Omega=17.6, \epsilon=0.068, t=430 \tau_{v}$. Lateral temperature boundary conditions are conducting. The gray denotes the conduction value $(\theta=0)$, and the lighter and darker shades give the values above and below this, which range from $\theta=-0.15$ to 0.15 .

the motion of a front, i.e., the boundary between the two different regions of roll orientation. Except for the special case of a front perpendicular to the rolls, the velocity of propagation of the front scales as $\epsilon^{1 / 2}$ [12], and the pertinent length scale varies as $\epsilon^{-1 / 2}$. This leads to the time scale for

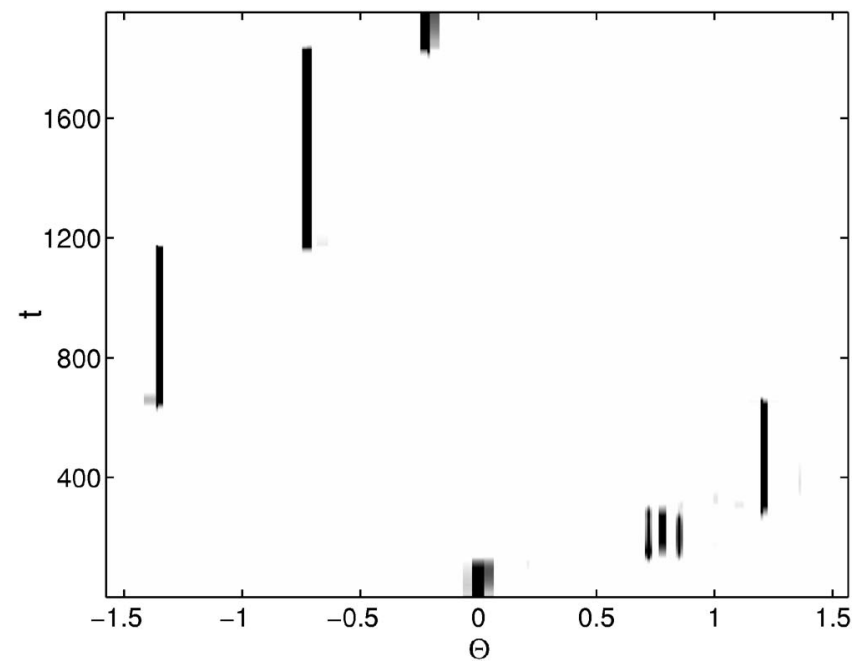

FIG. 2. A density plot of $F(\Theta, t)$ as a function of orientation angle $\Theta$ and time $t$. Black corresponds to the largest value, and white to the smallest. Because the angle of the rolls is a director field, only angles from $-\pi / 2$ to $\pi / 2$ are shown. Note that $-\pi / 2$ maps on to $\pi / 2$. The following parameters were used: $\Gamma=40, \sigma$ $=0.93, \Omega=17.6, \epsilon=0.007$, periodic boundaries. We cannot measure an $f_{\text {pre }}$ for this $\epsilon$ because the slope of the line through successive maxima increases with time.

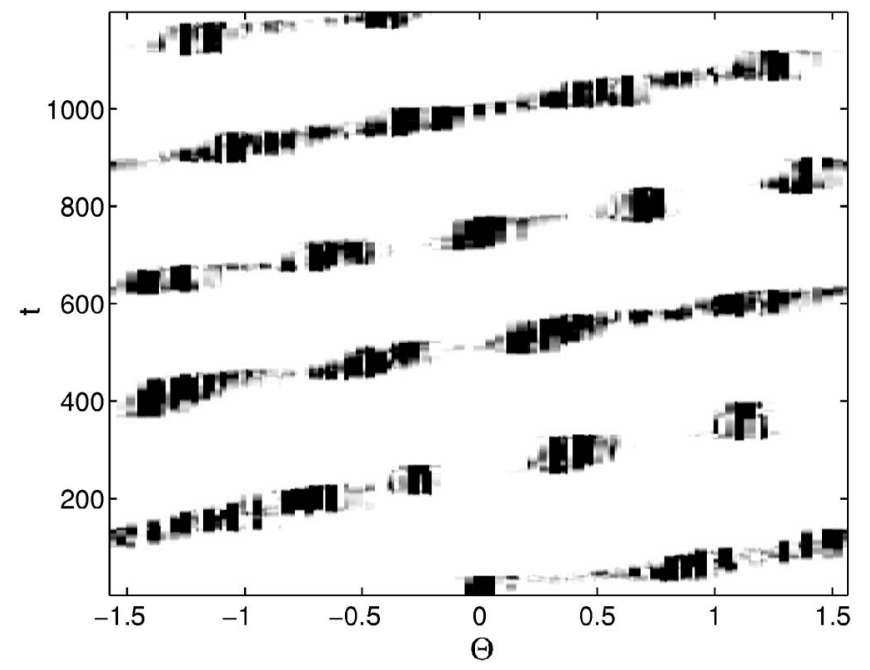

FIG. 3. The same type of plot as in Fig. 2 except $\epsilon=0.045$. The inverse of the slope of the dark line made from the dominant $\Theta$ as a function of time gives the precession frequency, which is $0.0126 \mathrm{rad}$ per vertical diffusion time in this case.

perturbations to grow, which scales as $\epsilon^{-1}$. These scaling predictions are some of the first quantitative, theoretical predictions for a spatiotemporally chaotic state.

Extensive experiments were done on cylindrical rotating Rayleigh-Bénard cells for aspect ratios 20 and 40 [13-16]. The scaling laws for characteristic lengths and times were found to differ from the theoretical predictions. Experimenters measured the scaling exponent for lengths to be -0.2 and times to be -0.6 , if they assumed that the length and time quantities diverged at onset [14]. However, the data could also be fitted by assuming no divergence at onset.

Our numerical code enables us to simulate periodic boundary conditions, as shown in Fig. 4, which more closely resemble the theoretical model. We can also simulate boundaries, as shown in Fig. 1, which closely resemble the experiment. By analyzing both types of geometries, we can determine the effect, if any, of realistic boundaries. Numerical analysis of a model equation had already indicated that realistic boundaries may play a role in the discrepancy between theory and experiment [17]. These boundaries should give rise to more complex spatial structure than periodic boundaries. This may have a stronger effect on the dynamics near onset.

\section{BOUSSINESQ EQUATIONS}

The system is modeled by the Boussinesq equations augmented by a Coriolis force [1]. The Boussinesq equations consist of a modified Navier-Stokes equation (1), the heat equation (2), and incompressibility (3). The equations are

$$
\begin{gathered}
\sigma^{-1}\left(\partial_{t}+\vec{u} \cdot \vec{\nabla}\right) \vec{u}=-\vec{\nabla} P+\nabla^{2} \vec{u}+\theta \hat{z}+2 \Omega \vec{u} \times \hat{z}, \\
\left(\partial_{t}+\vec{u} \cdot \vec{\nabla}\right) \theta=\nabla^{2} \theta+R w, \\
\vec{\nabla} \cdot \vec{u}=0 .
\end{gathered}
$$




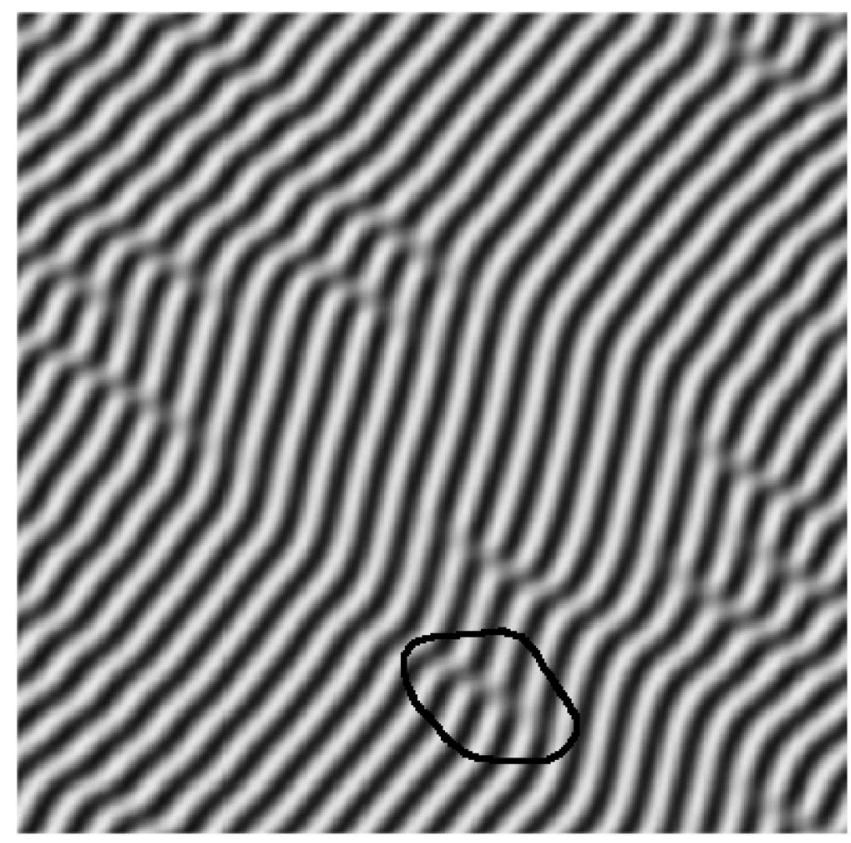

FIG. 4. Snapshot of temperature deviation $\theta$ at the midplane, for a domain chaos state with the following parameters: $\Gamma=40, \sigma$ $=0.93, \Omega=17.6, \epsilon=0.045, t=214 \tau_{v}$. Lateral temperature boundary conditions are periodic. The gray denotes the conduction value $(\theta$ $=0$ ), and the lighter and darker shades give the values above and below this, which range from $\theta=-0.12$ to 0.12 . A representative dislocation is circled.

The variables are nondimensionalized by specifying the length in terms of the cell height $d$, the temperature in terms of $\Delta T$, and the time in units of the vertical thermal diffusion time $\tau_{v}=d^{2} / \kappa$, where $\kappa$ is the thermal diffusivity. The variable $\vec{u}(\vec{r}, t)$ is the velocity field, $P(\vec{r}, t)$ is the pressure, and $\theta(\vec{r}, t)$ is the temperature deviation from the linear conduction profile. The symbol $\partial_{t}$ indicates time differentiation, and $\hat{z}$ is a unit vector in the vertical direction. The Prandtl number $\sigma=\nu / \kappa$, where $\nu$ is the kinematic viscosity. The Rayleigh number $R=\alpha g \Delta T d^{3} / \kappa \nu$, where $\alpha$ is the thermal expansion coefficient and $g$ is the acceleration of gravity. The variable $\Omega$ is the dimensionless rotation rate $\left(\Omega=\Omega_{D} d^{2} / \nu\right)$. The centrifugal force has been neglected because it is assumed to be small relative to the gravitational force $\left(\Omega_{D}^{2} r / g \ll 1\right)$ for our rotation rates. ${ }^{2}$

The aspect ratio $\Gamma$ is defined as the ratio of radius to depth for cylindrical regions and as the ratio of width to depth for periodic regions. The control parameter $\epsilon$ is defined as

$$
\epsilon=\frac{R-R_{c}}{R_{c}}
$$

where $R_{c}$ is the critical Rayleigh number at which conduction gives way to convection.

\footnotetext{
${ }^{2}$ There is recent evidence, both numerically and experimentally, that the centrifugal force may play a larger role than previously thought. This work is currently in preparation.
}

Along the top and bottom plates we use no-slip velocity boundary conditions and constant values for the temperature boundary conditions:

$$
\begin{gathered}
\vec{u}=\overrightarrow{0}, \quad \theta=1 / 2 \quad \text { at } z=0, \\
\vec{u}=\overrightarrow{0}, \quad \theta=-1 / 2 \quad \text { at } z=1 .
\end{gathered}
$$

For realistic boundary conditions on the sidewalls we use no-slip velocity boundary conditions and conducting thermal boundaries:

$$
\vec{u}=\overrightarrow{0}, \quad \theta=1 / 2-z \quad \text { at } r=\Gamma \quad \text { (conducting boundaries). }
$$

We will also use periodic (for square regions only, of course) boundary conditions on the sidewalls:

$$
\begin{gathered}
\vec{u}(x+\Gamma, y)=\vec{u}(x, y), \quad \theta(x+\Gamma, y)=\theta(x, y), \\
\vec{u}(x, y+\Gamma)=\vec{u}(x, y), \\
\theta(x, y+\Gamma)=\theta(x, y), \quad \text { (periodic boundaries). }
\end{gathered}
$$

To solve the Boussinesq equations with rotation, we have used the code NEK5000, a highly efficient, parallel, spectral element code developed to solve the Navier-Stokes equation. The details of the code are described elsewhere [18]. A fast code was essential because we ran our simulations out to 800 vertical diffusion times or longer for each $\epsilon$, in order to obtain good accuracy. As a result, our raw data are accurate to $1 \%$ or better. We used a spatial resolution of 0.1 and a time step of 0.005 . We used straight, parallel rolls as initial conditions for our simulations with conducting boundary conditions, and straight parallel rolls with a dislocation pair for our simulations with periodic boundary conditions. We used the dislocation pair to study the motion of defects in the periodic systems in more detail.

In order to determine scaling laws, the critical point, i.e., the point where $\epsilon \rightarrow 0$, must be determined precisely. In the spirit of experiments, we used our heat transport measurements to determine the critical Rayleigh number $R_{c}$. The Nusselt number is defined as the ratio of the total heat transported across the cell divided by the heat transported via conduction only. Hence if the Nusselt number is 1, there is only conduction, and if the Nusselt number is greater than one, there is convection as well. To allow for a simpler comparison, the reduced Nusselt number is studied: this is the Nusselt number minus 1 , which goes through zero at the onset of convection.

We determined the average reduced Nusselt number versus $R$ for our simulations of both periodic and conducting boundaries. From the quadratic fits shown we find $R_{c}$ (i.e., the $x$ intercept) to be 2246 for the periodic case and 2247 for the conducting case (see Fig. 5). If we instead perform a linear extrapolation through the first two data points, we find $R_{c}$ is 2248 for both cases. Our own results from linear stability analysis, along with Chandrasehkar's results [19] give a critical Rayleigh number of 2248. Since we would expect the effect of finite size to increase the critical Rayleigh num- 


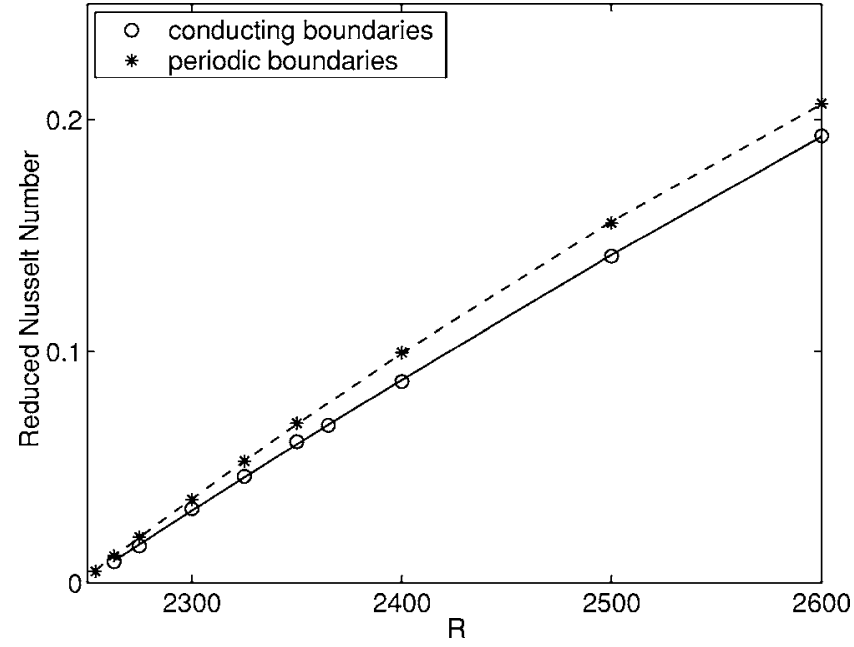

FIG. 5. Reduced Nusselt number versus Rayleigh number $(R)$ for both conducting and periodic boundaries. The following parameters were used in both cases: $\Gamma=40, \sigma=0.93, \Omega=17.6$. The solid line is a quadratic fit to the conducting boundary data and the dashed line is a quadratic fit to the periodic boundary data.

ber, we choose to use our linear extrapolation results of $R_{c}$ $=2248$. We find if we use the values from our quadratic fits instead, our scaling exponents change by at most $5 \%$.

\section{PRECESSION FREQUENCY}

One finds that the orientation of the rolls precesses (not necessarily smoothly) with time, in a counterclockwise direction for positive $\Omega$, as observed in the rotating frame. The precession frequency provides us with a good diagnostic quantity for time scaling. The precession frequency $f_{\text {pre }}$ is measured by first determining the dominant orientation angle $\Theta$ as a function of time. To obtain this quantity we first find the square of the modulus of the Fourier transform of the midplane temperature field $F(\vec{k})$ as a function of wave number $\vec{k}$ at one instant in time $t$. A radial average of this quantity gives us $F(\Theta)$, i.e., the Fourier power as a function of orientation angle. Then we can find this quantity for each time slice to give us $F(\Theta, t)$. Angle-time plots from representative cases are shown in Figs. 2 and 3. The rate of change of the orientation angle of the maximum in $F(\Theta, t)$ with respect to time gives the precession frequency.

Another quantity that can be measured is the domain switching frequency, $\tau_{\mathrm{dsw}}^{-1}$. According to the theory for rotating Rayleigh-Bénard convection [9], domains of straight parallel rolls become unstable to rolls at a different orientation. At threshold $(\epsilon=0)$, there is one distinct orientation and for $\epsilon>0$ there exists a band of orientation angles. This instability will cause the rolls to precess in a discrete manner. We can see this switching in Figs. 2 and 3, by noticing that the upwards sloping trend of the maximal value of $F(\Theta, t)$ often has discrete jumps. One can measure how long it takes for one set of rolls to become unstable to another set of rolls. The inverse of this transition time is known as the domain switching frequency. We find $\tau_{\mathrm{dsw}}^{-1}$ by first taking the autocorrelation function of the angle-time plot (see Fig. 6). Then the

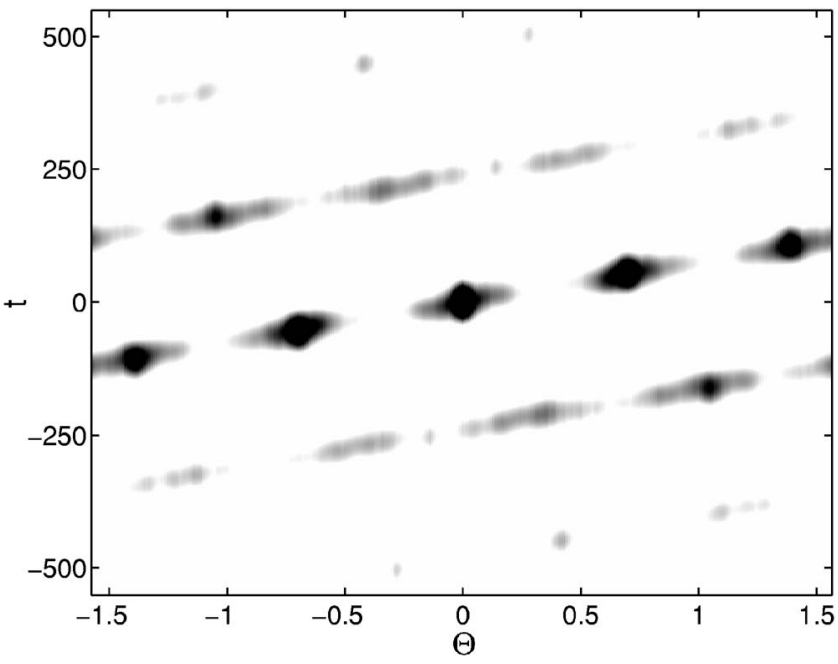

FIG. 6. The two-dimensional autocorrelation function of the angle-time plot shown in Fig. 3 (where the first $100 \tau_{v}$ were removed before taking the autocorrelation, to allow the transients to die out). The time between successive peaks is $55.5 \tau_{v}$; hence $f_{\mathrm{dsw}}$ is 0.018 . The switching angle $\theta_{\mathrm{sw}}$ is the difference in angle between successive peaks, and is $0.7 \pm 0.05$ for this case.

inverse of the time difference between the first peak (on either side) and the central peak gives the domain switching frequency. We can also find the domain switching angle $\theta_{\mathrm{sw}}$ by finding the difference in angle between the first peak and the central peak. We can then convert to the same units as $f_{\text {pre }}$ by multiplying $\tau_{\text {dsw }}^{-1}$ by $\theta_{\text {sw }}$ :

$$
f_{\mathrm{dsw}}=\tau_{\mathrm{dsw}}^{-1} \theta_{\mathrm{sw}} \text {. }
$$

The two precession frequencies $f_{\text {pre }}$ and $f_{\text {dsw }}$ should agree if the dominant method of precession is due to domain switching.

The domain switching angle $\theta_{\text {sw }}$ is plotted as a function of $\epsilon$ for both periodic and realistic boundary conditions in Fig. 7. The theoretical value for the maximum growth rate for this Prandtl number $(\sigma \approx 1)$ is about $0.7 \mathrm{rad}$ [20]. Our results are in good agreement with this value. Note however, that this is in disagreement with the experiments [14], which measured a switching angle of about $1.0 \mathrm{rad}$, and found a slight decrease in angle with $\epsilon$.

The quantities $f_{\text {pre }}$ and $f_{\text {dsw }}$ are plotted versus $\epsilon$ in Fig. 8 on a log-log scale for periodic and conducting boundaries. The slope of these lines gives the scaling of these frequency quantities with $\epsilon$. The scaling for both periodic and conducting boundary conditions does agree reasonably well with the theoretical results of timelike quantities scaling as $\epsilon^{-1}$. The average of all the slopes is 1.12 . We find that we can obtain a closer agreement with theory by noting that $\theta_{\text {sw }}$ also has a slight $\epsilon$ dependence, as can be seen in Fig. 7. Hence if we instead look at inverse transition times by dividing each of our $f_{\text {pre }}$ by its corresponding $\theta_{\mathrm{sw}}$, we can remove this slight bias:

$$
\tau_{\text {pre }}^{-1}=\frac{f_{\text {pre }}}{\theta_{\text {sw }}} .
$$

This is done in Fig. 9. Now we find that the average of all the slopes is 1.06. The theory [9] predicts that the dominant 


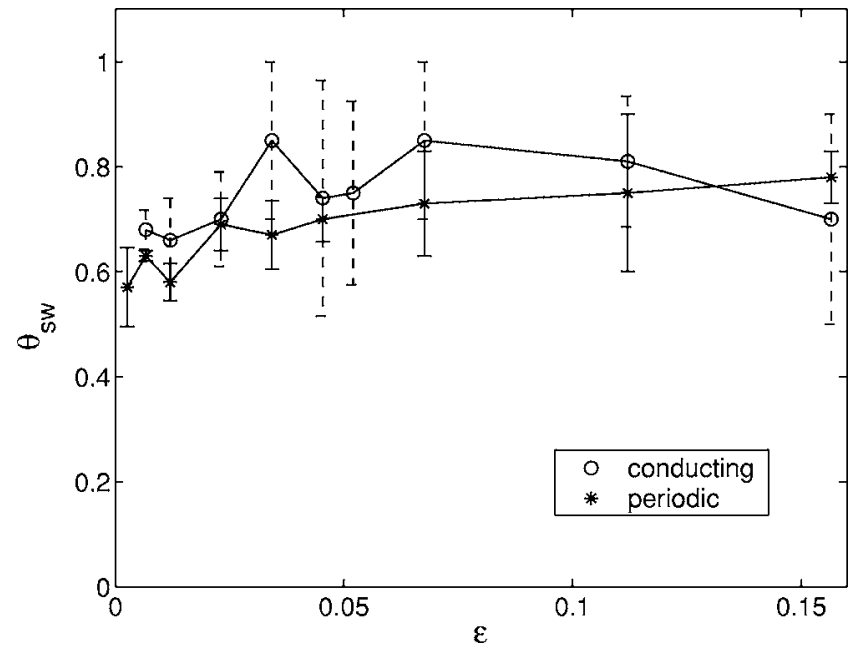

FIG. 7. Domain switching angle $\theta_{\mathrm{sw}}$ versus $\epsilon$ for both periodic and conducting boundaries for the same parameters as in Fig. 8. Note the slight $\epsilon$ dependence of $\theta_{\mathrm{sw}}$. The error bars (dashed for conducting and solid for periodic) are included to indicate the spread in angle on the autocorrelation plot (see Fig. 6 for an example).

method for precession is due to domain switching events. We see that our $\tau_{\text {pre }}^{-1}$ and $\tau_{\mathrm{dsw}}^{-1}$ very nearly coincide and that our scaling is consistent with theory. Hence one can conclude that predictions of the theoretical model for domain switching are consistent with the numerics, which are simulations of a realistic ideal Rayleigh-Bénard convection experiment.

However, the experimental results do not agree as seen in Fig. 10. We only plot $f_{\text {pre, }}$ and in units of rad per vertical diffusion time, to compare with the experimental results [14]. Note that the experimental results are for exactly the same parameters as the numerics for conducting boundaries, so it is somewhat surprising to find the precession frequencies differ significantly in magnitude. One also sees that scaling

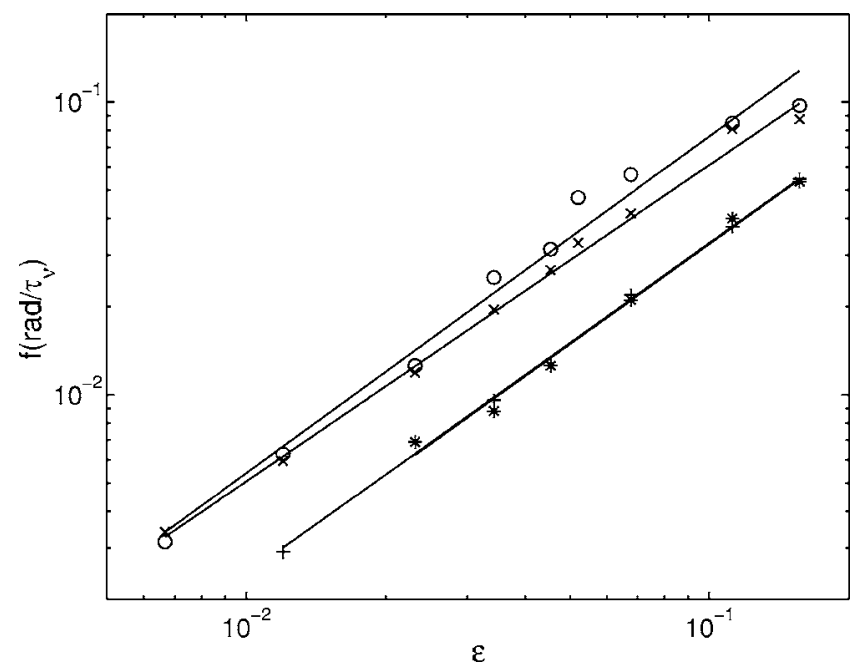

FIG. 8. Frequencies $f_{\text {pre }}$ and $f_{\text {dsw }}$ (in units of rad per vertical diffusion time) versus control parameter $\epsilon$. Legend: Conducting boundaries, $\bigcirc=f_{\text {pre }}$, slope $=1.15, \times=f_{\text {dsw }}$, slope $=1.08$. Periodic boundaries, $*=f_{\text {pre }}$, slope $=1.13,+=f_{\mathrm{dsw}}$, slope $=1.13$. The following parameters were used: $\Gamma=40, \sigma=0.93, \Omega=17.6$.

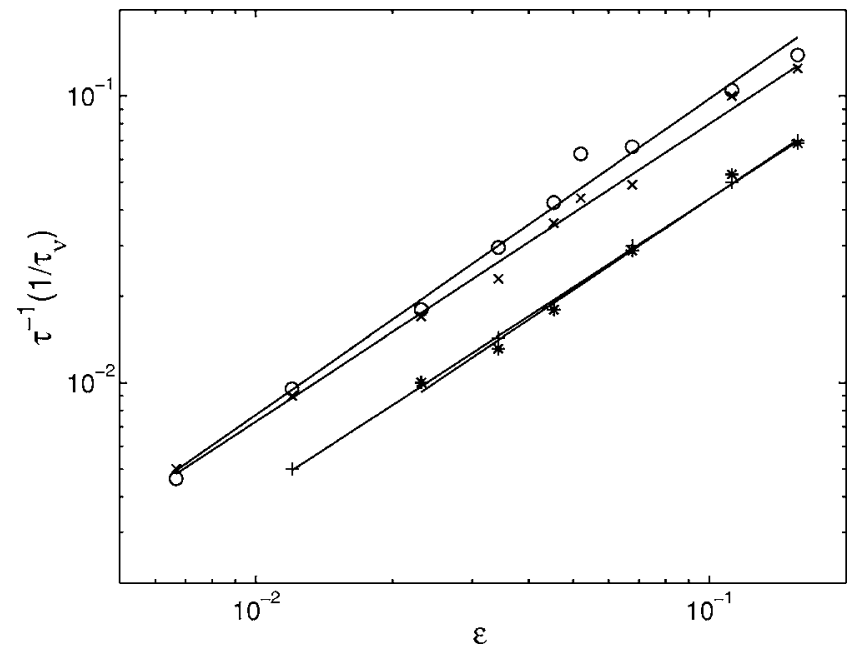

FIG. 9. Frequencies $\tau_{\text {pre }}^{-1}$ and $\tau_{\mathrm{dsw}}^{-1}$ (in units of inverse vertical diffusion times) versus control parameter $\epsilon$ for the same parameters as in Fig. 8. Legend: Conducting boundaries, $\bigcirc=\tau_{\text {pre }}^{-1}$, slope $=1.10$, $\times=\tau_{\mathrm{dsw}}^{-1}$, slope $=1.04$. Periodic boundaries, $*=\tau_{\text {pre }}^{-1}$, slope $=1.06,+$ $=\tau_{\mathrm{dsw}}^{-1}$, slope $=1.03$.

laws differ: the experiments find a slope of 0.58 whereas the theoretical slopes average to 1.1. However, if one looks only at the latter data points for the numerical conducting boundary case, one finds a slope of 0.68 , which is in better agreement with the experiment. This leads us to surmise that the experiments may not be measuring the theoretically predicted precession via domain switching, which should dominate for small enough $\epsilon$.

In addition, we have verified that there is another mechanism for precession, namely that of the gliding motion of defects (see Sec. V). This phenomenon can be explained by looking at a multiple scales perturbation expansion of the Swift-Hohenberg equation with rotation (see the Appendix). The new term due to the Coriolis force (A6) will cause dis-

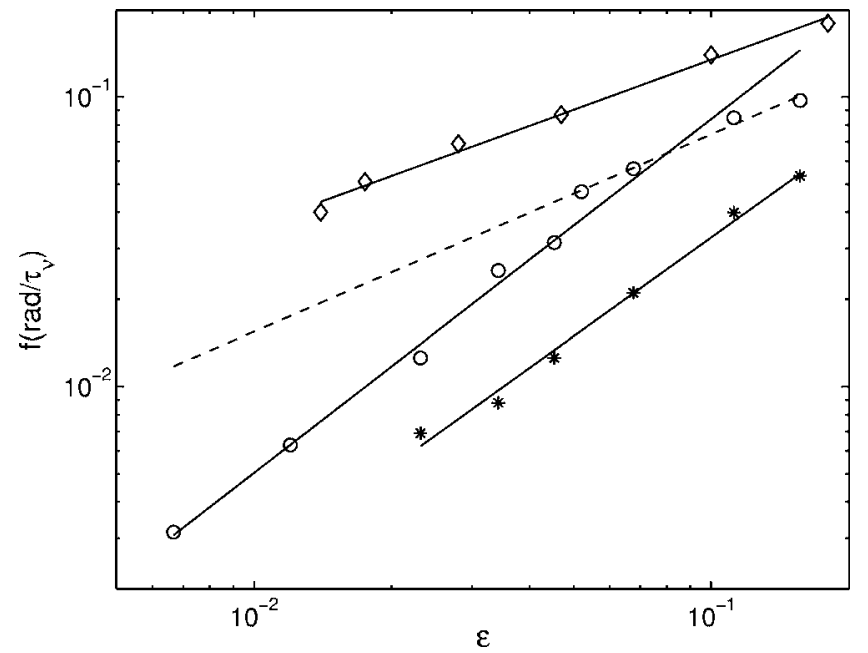

FIG. 10. Frequencies $f_{\text {pre }}$ (in units of rad per vertical diffusion time) versus control parameter $\epsilon$ for the same parameters as in Fig. 8. Legend: $\bigcirc=$ conducting boundaries, slope for early data points $=1.22$, slope for latter data points $=0.68$ (dashed line), $*=$ periodic boundaries, slope $=1.13, \diamond=$ experimental results, slope $=0.58$ [14]. 
location pairs which are stationary in a nonrotating system to glide in a rotating system, perpendicular to the rolls and in opposite directions. Their gliding path will cause an overall orientation change of the rolls due to the roll pinch off that occurs as they glide [21,22]. If one pair of dislocations is created in the center of a periodic cell, by the time they transverse the system, they will have caused the rolls to reorient by two roll diameters divided by the box length.

Even more generally, precession will occur whenever there is a gradient in the amplitude in the direction parallel to the rolls [23]. The precession will cause straight parallel rolls (whose amplitude goes to zero at a lateral boundary, for example) to bend and curve, ultimately creating dislocation pairs to release the stress. Dislocations will also appear if a domain switching event does not cleanly switch between one set of parallel rolls and another. This happens more often for higher $\epsilon$, where domains are smaller. This always happens for conducting boundaries, where the rolls can never fit perfectly. Dislocations can also be injected from sidewalls, and be created in regions of high curvature resulting from mean flows.

We expect the glide-induced precession frequency to scale differently with $\epsilon$ than precession via domain switching. We predict the glide-induced precession frequency $f_{\text {glide }}$ will scale as

$$
f_{\text {glide }} \propto \rho_{d} v_{\text {glide }}
$$

where $\rho_{d}$ is the linear density of defects and $v_{\text {glide }}$ is the glide velocity. We have found (see Sec. V again) the glide velocity to scale as $\epsilon^{3 / 4}$. If the density of defects remains relatively constant, then the glide-induced precession frequency should also scale with an exponent of 0.75 . This agrees better with the experimental slope of 0.58 and our own conducting boundary slope at larger $\epsilon$ of 0.68 .

As support for our reasoning, note that Busse and Heikes also performed measurements on rotating Rayleigh-Bénard convection $[6,24]$. They used water as the fluid, and measured the transition time, i.e., the time between domain switches. They found this transition time scaled with an exponent of -0.75 . They were further from threshold than the experiments of $\mathrm{Hu}$ et al. [14], but this scaling law supports our hypothesis of glide-induced precession being the dominant mode of precession at larger $\epsilon$.

\section{CORRELATION LENGTH}

For completeness, we have measured correlation lengths in the usual manner [25], even though recent work by Becker and Ahlers [26] have shown that this method is somewhat problematic. The basic idea is to measure the decay length of the azimuthally averaged autocorrelation function of the temperature field. This decay length gives the size over which domains are correlated, hence it is known as the correlation length. We accomplish this measurement by taking the Fourier transform of the midplane temperature field, finding the square of the modulus of this data, and then performing an azimuthal average. This is known as the structure function, $S(k)$, which is a measure of the wave-number distribution. We then average this structure factor over all of our

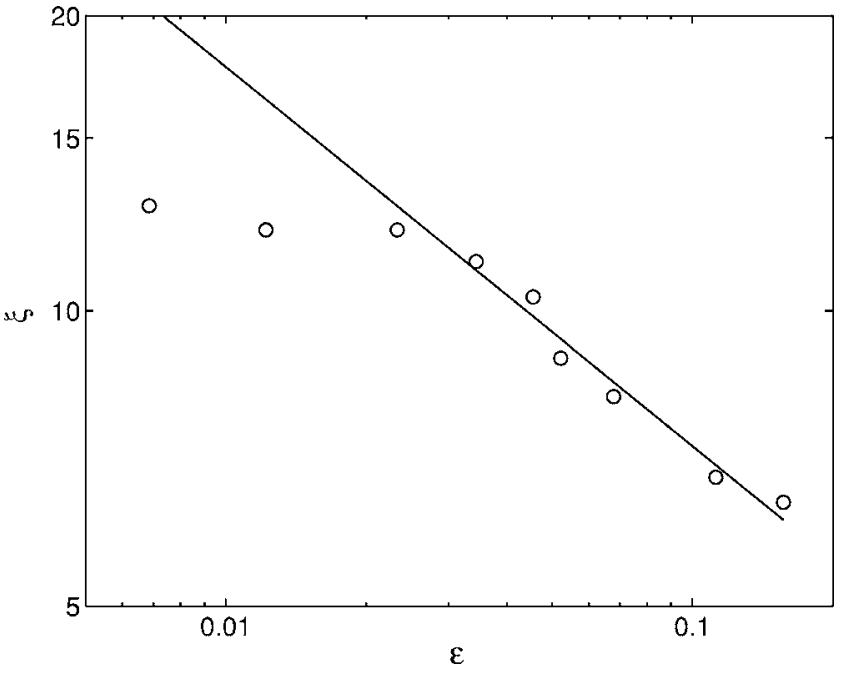

FIG. 11. Correlation length $\xi$ versus control parameter $\epsilon$ for conducting boundaries for the same parameters as in Fig. 8. The fit is to the last six data points, whose slope is -0.39 .

time slices from 100 vertical diffusion times and up. Next we fit the peak in this averaged structure function to a squared Lorentzian function. The inverse of the half-width is the correlation length $\xi$. It turns out that this procedure is rather sensitive to the fitting function: a Gaussian can yield different values, as does a simpler calculation of the second moment of the structure function. However, we find that the squared Lorentzian best models the data.

The results are shown in Fig. 11. Due to the finite size of the conducting cell, one is unable to measure a diverging correlation length for very small $\epsilon$. Eventually the rolls fill almost the entire cell, and the correlation length is determined by the aspect ratio, hence it levels off. As a result, our fit is only to the region where the correlation length begins to drop; which is the last six data points. For the periodic case, the correlation length is too large to measure accurately at any of our control parameters, so we do not show these results. The exponent in the conducting case of -0.39 is a bit smaller than the theoretical prediction of lengths scaling as $\epsilon^{-1 / 2}$, but is still in better agreement with the theory than the experiment. The experimental results find an exponent of -0.2 .

\section{DISLOCATIONS}

Dislocations are present in our simulations. An example is circled in Fig. 4. Dislocations that are stationary in a nonrotating system will glide in a direction perpendicular to the rolls (parallel to the roll wave vector) in the rotating system. An extensive analysis of defect velocities indicates that the dominant defect motion is perpendicular to the rolls for both our periodic and our realistic systems. The results can be seen for the periodic case in Fig. 12 and for the conducting case in Fig. 13. The dominant orientation angle of the rolls, $\Theta$, is plotted versus the angle of the velocity of the defects, $\theta_{v}$. We determined the dominant $\Theta$ by finding the $\Theta$ value corresponding to the maximum value of $F(\Theta)$ (such as in 


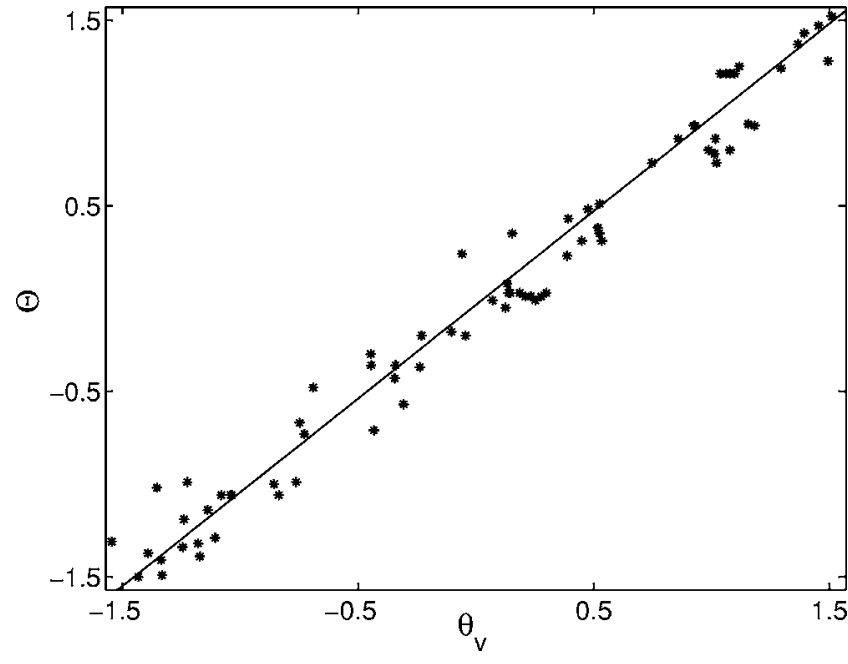

FIG. 12. Angle of the rolls, $\Theta$, versus angle of the velocity of defects, $\theta_{v}$, for periodic boundaries and the same parameters as in Fig. 8. The slope of the line is 1.01 , indicating that the defects mainly glide in a direction perpendicular to the rolls.

Fig. 3) at the instant in time when a defect is moving at its measured velocity. In both figures, the data is scattered about, but close to a line with unit slope passing through the origin. This does indeed prove that the dominant motion of our defects is glide, i.e., motion perpendicular to the rolls.

Our recently developed theoretical analysis has led to an understanding of the scaling of glide velocities with $\epsilon$ [23]. A brief derivation is given in the Appendix. One finds that

$$
v_{\text {glide }} \propto \epsilon^{3 / 4} \text {. }
$$

This is in excellent agreement with the numerical results in Fig. 14 for both the conducting and the periodic boundaries.

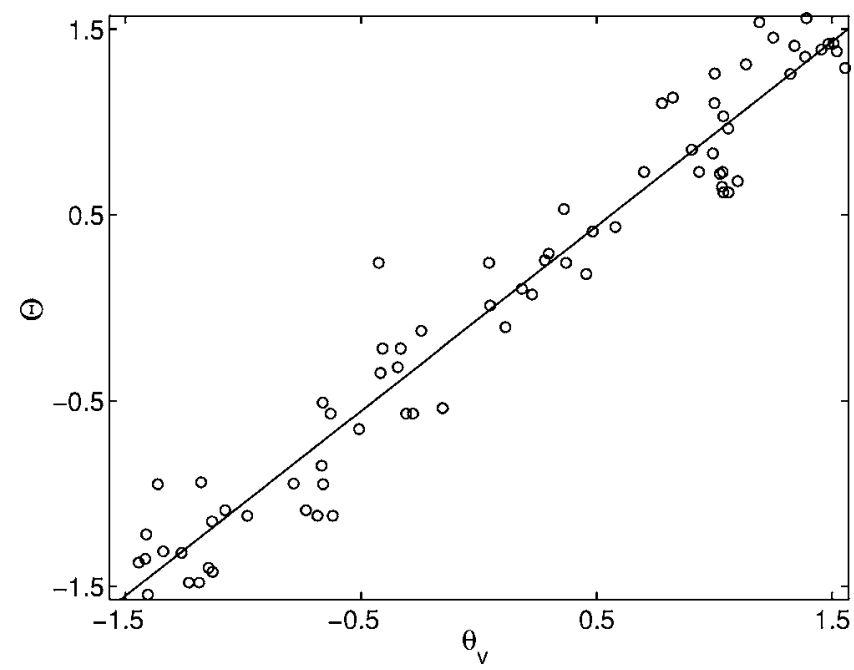

FIG. 13. The same type of plot as Fig. 12 but for conducting boundaries. The slope of the line is 0.98 , indicating that the defects mainly glide in a direction perpendicular to the rolls in the conducting case too. There is, however, more scatter than for the periodic case.

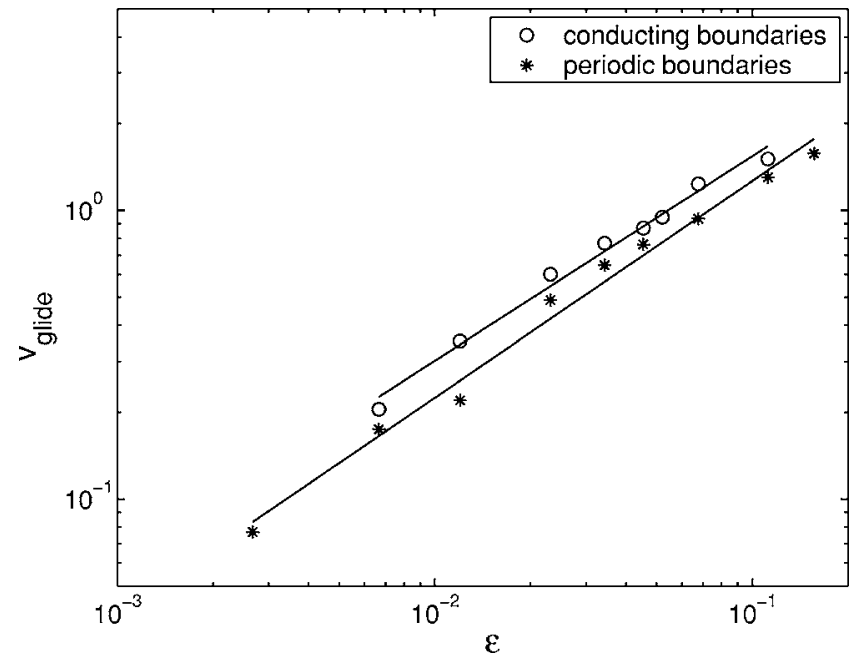

FIG. 14. Glide velocity of defects versus $\epsilon$ for the same parameters as in Fig. 8. Legend: $\bigcirc=$ conducting boundaries, slope $=0.71$, $*=$ periodic boundaries, slope $=0.75$. Approximately 150 defects were tracked, half for the conducting case, and half for the periodic case.

In the periodic case, one can separate out two different types of defect motion. One is the motion of isolated defect pairs and the other is the motion of defects during a domain switching event. As mentioned earlier, in a domain switching event, one set of rolls is replaced by another through the motion of a front. On the boundary between the two sets of rolls there is a superposition of both sets. This superposition creates a line of minima and maxima along the front. It is the motion of these minima that we track and call "domain switching defects."

We have plotted both types of defect motion in Fig. 15. (Note that the glide velocities shown in Fig. 14 for periodic boundaries are the isolated defects.) We find the domain switching defects lead to larger glide velocities, but they

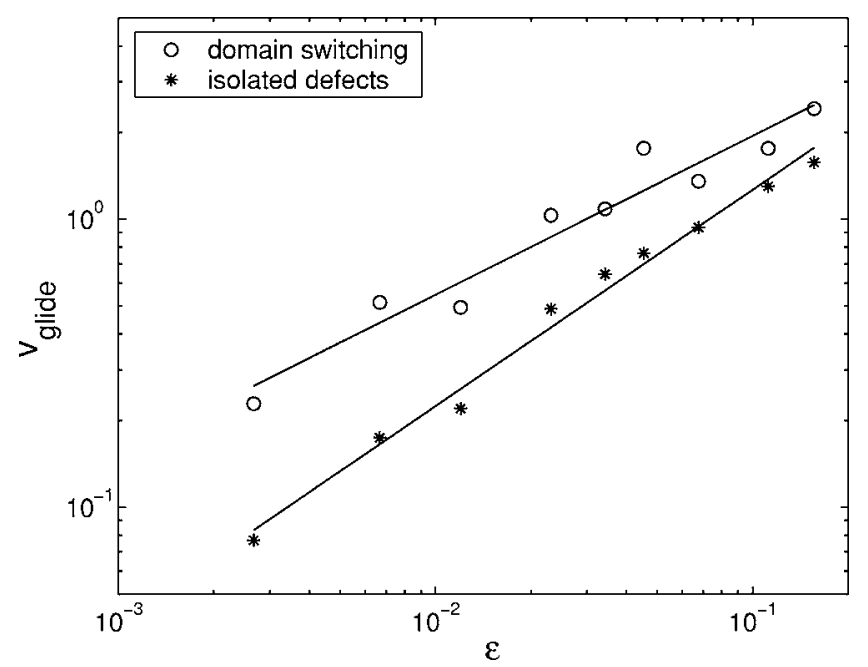

FIG. 15. Comparison of glide velocities from isolated defects and domain switching defects for the same parameters as in Fig. 8, but for periodic boundaries only. Legend: $\bigcirc=$ domain switching defects, slope $=0.55, *=$ isolated defects, slope $=0.75$. 


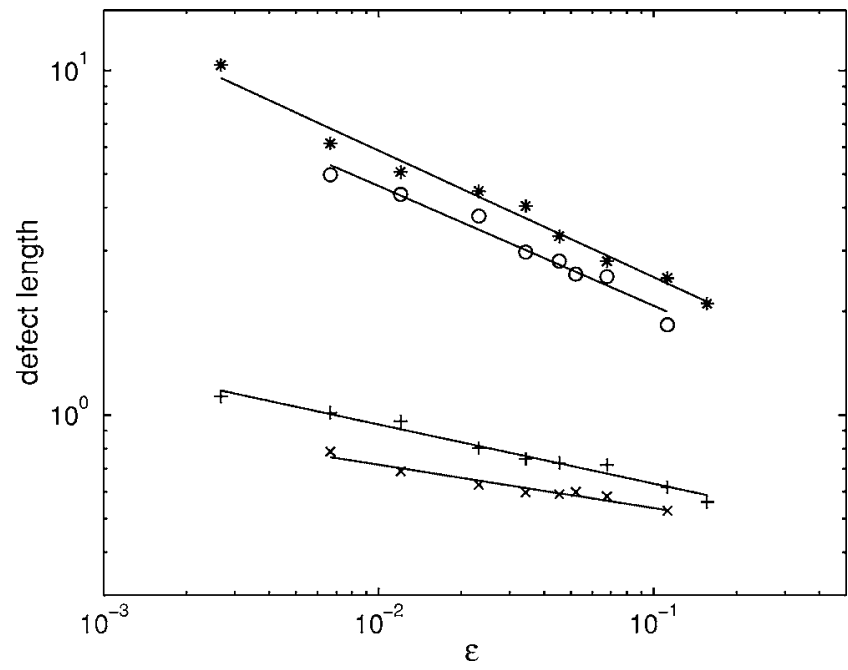

FIG. 16. Lengths of defects versus $\epsilon$ for the same parameters as in Fig. 8. Legend: Conducting boundaries, $\bigcirc=$ length of long axis, slope $=-0.35, \times=$ length of short axis, slope $=-0.13$. Periodic boundaries, $*=$ long axis, slope $=-0.37,+=$ short axis, slope $=-0.17$.

have an $\epsilon$ scaling exponent that is smaller (0.55) than the exponent for the isolated defects (0.75). We expect the velocity of the front to scale as $\epsilon^{0.5}$, which is in agreement with the results in Fig. 15. We could not clearly differentiate between these two types of defects in the conducting case, so the conducting data in Fig. 14 most likely contain both types of defects. This may explain the slightly smaller slope.

One can also measure the diameters of the axes of the defects, as shown in Fig. 16. Defects have a long and a short axis, and both were measured as a function of $\epsilon$ for periodic as well as conducting boundaries. The method of finding defect lengths is as follows. First we demodulated the midplane temperature field data, removing the underlying roll structure and only revealing the amplitude. Then we found the full width at half the height in the depression of the amplitude at the defect for both the long axis and the short axis of the defect. The multiple scale expansion (A2) predicts the long axis, perpendicular to the rolls, to scale as $\epsilon^{-1 / 2}$, and the short axis, parallel to the rolls, to scale as $\epsilon^{-1 / 4}$. In both the periodic and conducting cases, we find the long and short axes to scale with somewhat smaller exponents: the long axis scales with an average exponent of -0.36 , and the short axis with an average exponent of -0.15 . It is possible that we are unable to determine the correct scaling for the short axis, since we are measuring lengths smaller than a roll size. However, the long axis data should be correct. The long axis scaling does agree quite well with our correlation length scaling for conducting boundaries (see Fig. 11).

Next we turn to the scaling of defect areas. The theory predicts defect areas to scale as $\epsilon^{-3 / 4}$. If we compute defect areas by using the formula area $=\pi$ (long axis) (short axis) $/ 4$, our areas will scale as $\epsilon^{-0.51}$. We mention this since our numerical results do agree slightly better with preliminary experimental results [27]. It is unclear how to explain the discrepancy with theory.

\section{CONCLUSIONS}

Our numerical results for both periodic and conducting lateral temperature boundary conditions are in excellent agreement with the theoretical scaling laws for time as $\epsilon^{-1}$. We measured the precession frequency to scale with $\epsilon$ with an average exponent of 1.1 for periodic and conducting boundaries. Hence we find that time scales as $\epsilon^{-1.1}$. This is in disagreement with the experiments using the same parameters: they find that time scales as $\epsilon^{-0.6}$. It is possible that glide-induced precession may account for this discrepancy, since the theory assumes the precession of the roll orientation is entirely due to domain-switching events. The hypothesis of glide-induced precession with a constant dislocation density predicts precession frequencies to scale as $\epsilon^{0.75}$, which does agree better with the experiments.

We were unable to use correlation lengths to conclusively determine the length scaling for our numerical simulations with periodic boundary conditions, since the domains were simply too large. For our conducting boundary conditions we found correlation lengths to scale as $\epsilon^{-0.39}$ which is smaller than the theoretical prediction of $\epsilon^{-0.5}$, but is in worse disagreement with the experimental results of $\epsilon^{-0.2}$.

We did not chose to study the dependence of the scaling exponents on aspect ratio, since significantly larger aspect ratios are prohibitively expensive and one does not expect good scaling for the smaller ones. Earlier simulations on model equations [17] found little effect on correlation times for aspect ratios comparable to the ones we use, although the correlation length was significantly affected.

Our theoretical model for the motion of defects predicts that glide velocities will scale as $\epsilon^{0.75}$. Our numerical results confirm this. It is also interesting to note that for the conducting case, our length and time scales give a consistent velocity scaling: lengths (as $\epsilon^{-0.39}$ ) divided by times (as $\epsilon^{-1.1}$ ) gives velocities to scale as $\epsilon^{0.71}$. It would be very interesting for experimenters to also look at the scaling of velocities.

From our numerical results, we determined that defect areas scale on average as $\epsilon^{-0.51}$ for conducting boundaries and for periodic boundaries. This is in disagreement with the theory which predicts defect areas should scale as $\epsilon^{-0.75}$. In addition we looked at how the different axes in the defects scaled, and found the axis perpendicular to the rolls scaled as $\epsilon^{-0.36}$ and the axis parallel to the rolls scaled as $\epsilon^{-0.15}$, which clearly indicates there is a separation of scales in rotating Rayleigh-Bénard convection. However, the separation of scales is smaller than the predicted scaling of $\epsilon^{-0.5}$ and $\epsilon^{-0.25}$, respectively.

\section{ACKNOWLEDGMENTS}

We thank Paul Fischer for the use of his numerical code NEK5000, which was used for all of our simulations. We would like to thank Nathan Becker, Guenter Ahlers, Werner Pesch, Henry Greenside, Anand Jayaraman, Keng-Hwee Chiam, and Mark Paul for helpful discussions. This work was supported by the Engineering Research Program of the Office of Basic Energy Sciences at the Department of Energy, Grants No. DE-FG03-98ER14891 and No. DE-FG0298ER14892. The numerical code was run on the following 
supercomputing sites, whom we gratefully acknowledge: the National Computational Science Alliance under Grant No. DMR040001 which utilized the NCSA Xeon Linux Supercluster, the National Energy Research Scientific Computing Center which is supported by the Office of Science of the U.S. Department of Energy under Contract No. DE-AC0376SF00098, the Center for Computational Sciences at Oak Ridge National Laboratory, which is supported by the Office of Science of the Department of Energy under Contract No. DE-AC05-00OR22725, and "Jazz," a 350-node computing cluster operated by the Mathematics and Computer Science Division at Argonne National Laboratory as part of its Laboratory Computing Resource Center.

\section{APPENDIX: DERIVATION OF GLIDE VELOCITY}

For pedagogical reasons, we will discuss a multiple scale expansion of the Swift-Hohenberg equation with rotation [10], instead of the Boussinesq equations with rotation. The Swift-Hohenberg equation with rotation is

$$
\begin{aligned}
\partial_{t} \psi= & \epsilon \psi-\left(\nabla^{2}+q_{0}^{2}\right)^{2} \psi-g_{1} \psi^{3}+g_{2} \hat{z} \cdot \boldsymbol{\nabla} \times\left[(\nabla \psi)^{2} \boldsymbol{\nabla} \psi\right] \\
& +g_{3} \boldsymbol{\nabla} \cdot\left[(\boldsymbol{\nabla} \psi)^{2} \boldsymbol{\nabla} \psi\right] .
\end{aligned}
$$

The order parameter is $\psi, q_{0}$ is the wave number of the rolls, $g_{1}$ and $g_{3}$ are coefficients of the nonlinear terms, and $g_{2}$ is the coefficient of the term due to the Coriolis force, which is proportional to the rotation rate.

We will separate out fast $(x, z)$ and slow $\left(X, Y, T, T^{\prime}\right)$ scales in the following manner:

$$
\partial_{x} \rightarrow \partial_{x}+\epsilon^{1 / 2} \partial_{X}, \quad \partial_{y} \rightarrow \epsilon^{1 / 4} \partial_{Y}, \quad \partial_{t} \rightarrow \epsilon \partial_{T}+\epsilon^{5 / 4} \partial_{T^{\prime}}
$$

We will also expand $\psi$ in the small parameter $\epsilon$ :

$$
\psi=\epsilon^{1 / 2} \psi_{0}+\epsilon^{3 / 4} \psi_{1}+\epsilon \psi_{2}+\epsilon^{5 / 4} \psi_{3}+\epsilon^{3 / 2} \psi_{4}+\epsilon^{7 / 4} \psi_{5}
$$

We will assume $\psi=A e^{i q_{0} x}$, where the amplitude $A$ describes the overall modulation of the roll structure and hence it only depends on the slow variables $X, Y, T, T^{\prime}$. To second order, $A=\epsilon^{1 / 2} A_{0}+\epsilon^{3 / 4} A_{1}$.

This leads to the usual terms in the amplitude equation at order $\epsilon^{3 / 2}$ :

$$
\left[-\partial_{T}+1-\left(2 i q_{0} \partial_{X}+\partial_{Y}^{2}\right)^{2}-3\left(q_{0}^{4} g_{3}+g_{1}\right)\left|A_{0}\right|^{2}\right] A_{0}=0,
$$

or in shorthand notation,

$$
\Lambda\left[A_{0}\right]=0 .
$$

New rotation terms in enter into the amplitude equation at order $\epsilon^{7 / 4}$ :

$$
L A_{1}=\partial_{T^{\prime}} A_{0}+4 i q_{0}^{3} g_{2}\left(A_{0} \partial_{Y}\left|A_{0}\right|^{2}\right)
$$

where

$$
L \delta A_{0}=\Lambda\left[A_{0}+\delta A_{0}\right]-\Lambda\left[A_{0}\right]+O\left(\delta A_{0}^{2}\right)
$$

is the next order perturbation solution, and $A_{0}$ is the solution to Eq. (A5).

One can compute the glide velocity $v_{\text {glide }}$ from Eq. (A6), following [28-33]. First we assume $A_{0}=A_{d}$ is a stationary defect solution to Eq. (A5). Then we transform into a frame moving with the glide velocity, $v_{\text {glide }} \hat{X}$. This implies $A_{d}(X, Y) \rightarrow A_{d}\left(X-v_{\text {glide }} T^{\prime}, Y\right)$, and we find that Eq. (A6) becomes

$$
L A_{1}=4 i q_{0}^{3} g_{2}\left(A_{d} \partial_{Y}\left|A_{d}\right|^{2}\right)-v_{\text {glide }} \partial_{X} A_{d} .
$$

In order to solve this equation, we need to find the zero eigenvalue mode of $L^{\dagger}$, the adjoint of $L$. Since $L$ is selfadjoint, we can use the zero eigenvalue mode $\partial_{X} A_{d}$ of $L$, which corresponds to the translational symmetry of the dislocation perpendicular to the stripes. Hence the right-hand side of Eq. (A8) must be orthogonal to $\partial_{X} A_{d}$ :

$$
\left\langle\partial_{X} A_{d}, 4 i q_{0}^{3} g_{2}\left(A_{d} \partial_{Y}\left|A_{d}\right|^{2}\right)-v_{\text {glide }} \partial_{X} A_{d}\right\rangle=0,
$$

where

$$
\langle u, v\rangle=\int u^{\dagger} v d X d Y .
$$

This yields the following relationship for the glide velocity:

$$
v_{\text {glide }}=\frac{\left.4 i q_{0}^{3} g_{2}\left\langle\partial_{X} A_{d}, A_{d} \partial_{Y}\left|A_{d}\right|^{2}\right\rangle\right\rangle}{\left\langle\partial_{X} A_{d}, \partial_{X} A_{d}\right\rangle} .
$$

We can actually find a numerical value for $v_{\text {glide }}$ for a given $A_{d}$ solution. However, we are interested in the scaling here. Reverting back to unscaled variables using Eqs. (A2) and (A3) gives

$$
v_{\text {glide }} \propto \epsilon^{3 / 4} .
$$

Note that our glide velocity scaling with $\epsilon^{3 / 4}$ disagrees with the velocity scaling derived in [21]. They found the glide velocity to scale linearly with $\epsilon$ for small $\Omega$. However, they did not use the separation of scales (A2).
[1] M. C. Cross and P. C. Hohenberg, Rev. Mod. Phys. 65, 851 (1993).

[2] A. Schlüter, D. Lortz, and F. Busse, J. Fluid Mech. 23, 129 (1965).

[3] P. Manneville, Dissipative Structures and Weak Turbulence (Academic Press, New York, 1990).

[4] G. Küppers and D. Lortz, J. Fluid Mech. 35, 609 (1969).
[5] G. Küppers, Phys. Lett. 32A, 7 (1970).

[6] F. H. Busse and K. E. Heikes, Science 208, 173 (1980).

[7] D. Laveder, T. Passot, Y. Ponty, and P. L. Sulem, Phys. Rev. E 59, R4745 (1999).

[8] J. Oh and G. Ahlers, Phys. Rev. Lett. 91, 094501 (2003).

[9] Y. Tu and M. C. Cross, Phys. Rev. Lett. 69, 2515 (1992).

[10] M. C. Cross, D. Meiron, and Y. Tu, Chaos 4, 607 (1994). 
[11] E. Knobloch, Int. J. Eng. Sci. 36, 1421 (1998).

[12] E. Ben-Jacob, H. R. Brand, G. Dee, L. Kramer, and J. S. Langer, Physica D 14, 348 (1985).

[13] F. Zhong and R. E. Ecke, Chaos 2, 163 (1992).

[14] Y. Hu, R. E. Ecke, and G. Ahlers, Phys. Rev. Lett. 74, 5040 (1995).

[15] Y. Hu, R. E. Ecke, and G. Ahlers, Phys. Rev. E 55, 6928 (1997).

[16] Y. Hu, W. Pesch, G. Ahlers, and R. E. Ecke, Phys. Rev. E 58, 5821 (1998).

[17] M. C. Cross, M. Louie, and D. Meiron, Phys. Rev. E 63, 045201(R) (2001).

[18] P. F. Fischer, J. Comput. Phys. 133, 84 (1997).

[19] S. Chandrasekhar, Hydrodynamic and Hydromagnetic Stability (Oxford University Press, Oxford, 1961).

[20] T. Clune and E. Knobloch, Phys. Rev. E 47, 2536 (1993).

[21] J. Millán-Rodríguez, C. Pérez-García, M. Bestehorn, M. Neu- feld, and R. Friedrich, Chaos 4, 369 (1994).

[22] J. Millán-Rodríguez, M. Bestehorn, C. Pérez-García, R. Friedrich, and M. Neufeld, Phys. Rev. Lett. 74, 530 (1995).

[23] J. D. Scheel and M. C. Cross (unpublished).

[24] K. E. Heikes, Ph.D. thesis, University of California, Los Angeles, 1979 (unpublished).

[25] S. W. Morris, E. Bodenschatz, D. S. Cannell, and G. Ahlers, Phys. Rev. Lett. 71, 2026 (1993).

[26] N. Becker and G. Ahlers (private communication).

[27] N. Becker and G. Ahlers, private communication, 2004.

[28] G. Tesauro and M. C. Cross, Phys. Rev. A 34, 1363 (1986).

[29] E. D. Siggia and A. Zippelius, Phys. Rev. A 24, 1036 (1981).

[30] Y. Pomeau, S. Zaleski, and P. Manneville, Phys. Rev. A 27, 2710 (1983).

[31] K. Kawasaki, Prog. Theor. Phys. Suppl. 79, 161 (1984).

[32] K. Kawasaki, Prog. Theor. Phys. Suppl. 80, 123 (1984).

[33] H. Brand and K. Kawasaki, J. Phys. A 17, L095 (1984). 